\title{
O papel da Educação Permanente em Saúde na Atenção Primária e a pandemia de COVID-19
}

\author{
The role of Permanent Health Education in Primary Care and the \\ COVID-19 pandemic
}

\author{
Carolina Dutra Degli Esposti ${ }^{\top}$ \\ Lorena Ferreira ${ }^{1}$ \\ Ana Rosa Murad Szpilman ${ }^{2}$ \\ Marly Marques da Cruz 3
}

${ }^{1}$ Universidade Federal do Espírito Santo. Vitória/ES, Brasil.

${ }^{2}$ Universidade Vila Velha. Vila Velha/ES, Brasil.

${ }^{3}$ Fundação Oswaldo Cruz. Rio de Janeiro/ES, Brasil.

\begin{abstract}
A Educação Permanente em Saúde (EPS) é uma estratégia político-pedagógica de formação em saúde fundamentada em uma aprendizagem que se dá no e com relação ao próprio processo de trabalho, visando à transformação das práticas em saúde ${ }^{1,2}$. Sua introdução na América Latina, e consequentemente no Brasil, deu-se na década de 1980, por ação da Organização Pan-Americana da Saúde (OPAS) ${ }^{3}$, tendo se tornado uma política nacional a partir de 20044,5. Desde então, tem-se buscado a sua implementação em todo o território nacional para qualificação e desenvolvimento dos profissionais de saúde e, consequentemente, melhoria dos serviços prestados e fortalecimento do Sistema Único de Saúde (SUS).

Como modelo de atenção à saúde no Brasil, a Atenção Primária à Saúde (APS) atua como a porta de entrada para os serviços públicos de saúde e concentra grande força de trabalho em um processo contínuo de atenção aos cuidados essenciais à saúde da população. Nesse cenário, a EPS visa fortalecer as práticas em APS, e suas principais iniciativas relacionam-se ao desenvolvimento de recursos tecnológicos, à aproximação entre as instituições de ensino e saúde para garantir uma prática de ensino-serviço e à formação de espaços coletivos de aprendizagem significativa no cotidiano do trabalho. Contudo, existe, ainda, a desvalorização das iniciativas de EPS, o que se torna uma barreira à sua efetivação na APS $^{6}$.
\end{abstract}


Sendo a proposta da EPS uma ferramenta de transformação das práticas no trabalho, é preciso avançar na compreensão do conceito de EPS que rege a política e de suas práticas nos serviços pelos gestores, profissionais de saúde, instituições de ensino e usuários, a fim de que sejam aprimorados tanto a formação em saúde quanto os processos de trabalho no cotidiano dos serviços. Além disso, precisa se ajustar às novas demandas que se apresentam a esses serviços, inclusive aquelas decorrentes das mudanças nas situações epidemiológicas locais, que são reflexo das alterações nas necessidades de saúde dos indivíduos e das comunidades.

Destaca-se que, desde o início do ano de 2020, o mundo convive com a pandemia de COVID-19, impactando nos processos de trabalho em saúde. O primeiro caso de infecção pelo novo coronavírus (Severe Acute Respiratory 2-SARSCov-2) foi reportado na China, no início de dezembro de $2019^{7}$. Considerada uma doença de elevada transmissibilidade ${ }^{8}$, apresentou uma escalada acelerada em número de casos novos em esfera global, fazendo com que a Organização Mundial da Saúde (OMS) a considerasse uma pandemia.

Desde o início da pandemia até o final do mês de agosto de 2020, foram registrados aproximadamente 25 milhões de casos e 800 mil mortes pela doença em todo o mundo. Apesar do moderado declínio ou estabilização observados nas taxas de incidência na região das Américas, o Brasil permanece, assim como outros países da América Latina, no grupo de maior taxa de incidência a cada 1 milhão de habitantes, superior a 1.000 novos casos diários. Diante de tal situação, o país apresenta o expressivo acumulado de 3.804 .803 casos e 119.504 mortes?.

A COVID-19 é uma doença respiratória, cuja transmissão está associada à inalação ou ao contato com gotículas infectadas de pacientes com ou sem sintomas clínicos aparentes, eliminadas por meio de tosse ou espirro que podem alcançar um espaço de um a dois metros ${ }^{10}$. A elevada capacidade de reprodução do vírus causador contribui em sua transmissibilidade ${ }^{11}$, assim como o prolongado período de incubação, tempo compreendido entre a infecção do indivíduo e a apresentação de sintomas, que varia entre dois a onze dias, muito interfere na rápida disseminação da doença ${ }^{12}$.

No Brasil, a APS trabalha para garantir o acesso aos serviços de saúde com base no conhecimento do território, no vínculo entre o usuário e a equipe de saúde e na integralidade da assistência, e, nesse contexto, exerce papel fundamental no monitoramento das famílias vulneráveis e no acompanhamento aos casos suspeitos e leves. Dessa forma, a assistência na APS é estratégia fundamental tanto para a contenção da pandemia, quanto para o não agravamento das pessoas diagnosticadas com a COVID-19 ${ }^{13}$.

Além disso, na ausência de uma vacina para a COVID-19, foram tomadas outras medidas de saúde pública, denominadas intervenções não farmacêuticas, visando à redução do contato entre pessoas e da transmissão do vírus ${ }^{14}$. Dentre essas medidas, a que tem se mostrado mais efetiva é o distanciamento social, compulsório ou voluntário, dos indivíduos. No entanto, essa medida pode se apresentar como uma experiência desagradável para a população, uma vez que o isolamento, a perda de liberdade, as incertezas sobre o estado de saúde e o tédio podem provocar efeitos devastadores ${ }^{15}$.

Assim sendo, os serviços na APS também necessitam abordar os problemas relacionados ao isolamento social prolongado e à precarização da vida social e econômica, como transtornos mentais, violência doméstica, alcoolismo e agudização ou desenvolvimento de agravos crônicos, que coexistirão com as necessidades já apresentadas pelas pessoas e comunidades $^{13}$. As equipes de saúde precisaram ajustar o processo de cuidado aos usuários do sistema e enfrentar todas as necessidades advindas das inúmeras mudanças sociocomportamentais dentro das sociedades, incluindo alterações no estilo de vida, que se relacionam com o aumento do convívio familiar ${ }^{16}$ e alterações na rotina de práticas de atividades físicas ${ }^{16,17}$ e alimentares ${ }^{16}$. Além do comprometimento da saúde física, estudos demonstraram que a quarentena foi o fator mais preditivo dos sintomas de estresse agudo ${ }^{15}$, sendo desordens relacionadas à ansiedade, depressão e estresse póstraumático as principais manifestações ${ }^{18}$. Por fim, há um agravamento do cenário de adoecimento da população, gerado pela circulação de mitos e notícias falsas, principalmente em redes sociais e vídeos alarmantes ${ }^{19}$. 
O contexto da pandemia de COVID-19 trouxe como desafio aos profissionais de saúde nos serviços e na prestação do cuidado a necessidade de análises rápidas sobre a etiologia da doença, assim como suas formas de controle e prevenção, além do enfrentamento de todos os aspectos intervenientes na saúde mental e física da população por conta do cenário da pandemia. A grande velocidade com que os dados são divulgados e a existência de variadas fontes de informação apresentam-se como desafios para gestores, trabalhadores da saúde e usuários. Como lidar com tanta informação e em tão curto espaço de tempo? Como escolher as melhores fontes de informação?

Para o enfrentamento da pandemia de COVID-19 nos serviços de saúde, algumas iniciativas de EPS foram desenvolvidas no sentido da reorganização do processo de trabalho e da prestação do cuidado. No Centro da APS de um município do sul da Bahia, as ações de EPS foram direcionadas para discussões dos protocolos e documentos relacionados à COVID-19. No entanto, diante da necessidade de constante atualização imposta pela pandemia, com novos protocolos e novas orientações redefinidas quase que diariamente pela OMS e pelo Ministério da Saúde (MS), uma atitude construtiva foi desenvolvida pela equipe de saúde com a elaboração de um plano estratégico com ações de curto prazo, específicas a sua realidade; e a realização de uma triagem imediata no acesso do indivíduo à unidade, com o intuito tanto de diminuir aglomerações, quanto para identificar precocemente casos sintomáticos respiratórios, possibilitando seu isolamento. Em relação aos procedimentos, manteve-se a realização de curativo e fornecimento do material para sua realização em domicílio, além de ações educativas nas salas de espera sobre os temas higiene respiratória, distanciamento social e saúde mental ${ }^{20}$.

Também nesse contexto, é importante criar novos pontos de acesso ao sistema de saúde, especialmente por via remota. Por meio da modalidades de teleatendimento (teleorientação, teleconsulta, telemonitoramento e telerregulação), que passam a desempenhar papel central a partir desse momento, para os casos de pessoas com níveis de ansiedade elevados e/ou sintomas sugestivos de COVID-19 brandos ou com gravidade moderada, com possibilidade de orientar os casos suspeitos e o reconhecimento dos sinais de alerta; monitorar esses casos suspeitos quanto à evolução clínica; realizar vídeo consultas para casos mais complexos e solicitar remoção para uma unidade hospitalar ao identificar sinais de agravamento ${ }^{21,22}$.

Mudanças também foram impostas no arcabouço legal que rege a prática de telessaúde no país, o que levou à regulamentação, pelo MS, do uso de telemedicina, excepcionalmente para o contexto da pandemia de COVID-1923, com fins de prestação de abordagem pré-clínica, suporte assistencial, consulta, monitoramento e diagnóstico em todo o sistema de saúde brasileiro. Isso resultou, dentre outras coisas, na adoção, por estados e municípios, de centrais de teleatendimento como componentes essenciais de primeiro contato das pessoas com a rede de atenção à saúde ${ }^{13}$.

A reorganização do processo de trabalho das equipes e a autonomia dos profissionais de saúde, além de serem fundamentais no enfrentamento da pandemia, podem, também, amenizar os efeitos sociais e econômicos das medidas de distanciamento social na população. Por meio das mídias sociais e rádios comunitárias, as equipes podem orientar a população sobre as formas de contágio e informar sobre os canais de teleatendimento, além de ajudar a identificar indivíduos e famílias em situação de maior vulnerabilidade, mobilizando recursos da própria comunidade para auxiliar na entrega de alimentos e outros itens essenciais ${ }^{21}$.

Considera-se que a APS desempenha um papel central na mitigação dos efeitos da pandemia e, com o desenvolvimento de iniciativas de EPS, pode contribuir para diminuir a incidência da infecção na população adscrita, por meio do trabalho comunitário na redução da disseminação da infecção e no acompanhamento dos casos leves em isolamento domiciliar, na identificação das situações de vulnerabilidade individual ou coletiva e, principalmente, garantindo o acesso aos cuidados de saúde e o necessário encaminhamento nas fases mais críticas da epidemia, com impacto direto na diminuição da morbimortalidade. Mais uma vez, reafirma-se a EPS como uma ferramenta de transformação das práticas em saúde, que se dá no e com relação ao próprio processo de trabalho, e que deve envolver a gestão, os profissionais da saúde, as instituições de ensino e os usuários na busca por inovações e soluções tecnológicas para os desafios da saúde pública e da valorização da vida. 


\section{REFERÊNCIAS |}

1. Campos KFC, Sena RR, Silva KL. Educação permanente nos serviços de saúde. Esc. Anna Nery Rev. Enferm. 2017 ago; 2(4):1-10.

2. Silva JF. A educação permanente em saúde como espaço de produção de saberes na Estratégia de Saúde da Família [dissertação]. Rio de Janeiro: Escola Nacional de Saúde Pública Sergio Arouca; 2015. Disponível em: https://www.arca. fiocruz.br/bitstream/icict/13461/1/30.pdf.

3. Lemos CLS. Educação Permanente em Saúde no Brasil: educação ou gerenciamento permanente? Ciênc. Saúde Colet. [internet]. 2016; 21(3):913-922. Disponível em: http://www.scielo.br/scielo.php?pid=S1413=81232016000300913-\&scriptsci_abstract\&tlng=pt.

4. Brasil. Ministério da Saúde. Secretaria de Gestão do Trabalho e da Educação na Saúde. Política de Educação Permanente e Desenvolvimento para o SUS: caminhos para educação permanente em saúde. Brasília, DF: Ministério da Saúde; 2004. 68 p. (Série C. Projetos, Programas e Relatórios).

5. Brasil. Ministério da Saúde. Secretaria de Gestão do Trabalho e da Educação na Saúde. Departamento de Gestão da Educação em Saúde. Política Nacional de Educação Permanente em Saúde. Brasília, DF: Ministério da Saúde, 2009. 64 p. (Série B. Textos Básicos de Saúde) (Série Pactos pela Saúde 2006; v. 9).

6. Ferreira L, Barbosa JSA, Esposti CDD, Cruz MM. Educação Permanente em Saúde na atenção primária: uma revisão integrativa da literatura. Saúde debate [Internet]. 2019; 43(120):223-239. Disponível em: http://www.scielo.br/scielo. php?script=sci_arttext\&pid=S0103-11042019000100223\&lng=en.

7. Xiao C. A Novel Approach of Consultation on 2019 Novel Coronavírus (COVID-19) - Related Psychological and Mental Problems: structured letter therapy. Psychiatry Investigation. 2020; 17(2):175-176. Disponível em: https://www. psychiatryinvestigation.org/upload/pdf/pi-2020-0047.pdf.

8. Chaves TSS, Bellei NCJ. SARS-COV-2, o novo Coronavírus. Revista de Medicina. 2020; 99(1):1-4. Disponível em: https://www.revistas.usp.br/revistadc/article/view/167173/159662.

9. OMS. Coronavirus disease (COVID-19) Weekly Epidemiological Update. Disponível em: https://www.who.int/docs/ default-source/coronaviruse/situation-reports/20200831-weekly-epi-update-3.pdf?sfvrsn=d7032a2a_4.

10. Silva DP, Santos IMR, Melo VS. Aspectos da infecção ocasionada pelo Coronavírus da Síndrome Respiratória Aguda Grave 2 (SARS-CoV-2). Brazilian Journal Of Health Review. 2020; 3(2):3763-3779. Disponível em: http:/ /www.brazilianjournals.com/index.php/BJHR/article/view/9304/7858.

11. He Xi, Lau EHY, Wu P, Deng X, Wang J, Hao X, Lau YC, Wong JY, Guan Y, Tan X, Mo X, Chen Y, Liao, B, Chen W, Hu F, Zhang Q, Zhong M, Wu Y, Zhao L, Zhang F, Cowling BJ, Li F, Leung GM. Temporal dynamics in viral shedding and transmissibility of COVID-19. Nature Medicine. 2020; 26(5):672-675. Disponívelem: https://www.nature.com/articles/s41591-020-0869-5.pdf.

12. Backer J, Klinkenberg D, Wallinga J. Incubation period of 2019 novel coronavirus (2019-nCoV) infections among travellers from Wuhan, China, 20-28 January 2020. Eurosurveillance. 2020;25(5):1-6. Disponível em: https:/ /www.eurosurveillance.org/docserver/fulltext/eurosurveillance / 25/5/eurosurv-25-5-3.pdf?expires=1591040011\&id=id\&accnam $\mathrm{e}=$ guest\&checksum $=72$ C6CFCB7A5F74AB6BFEC35A974EBCC7. 
13. Sarti TD, Lazarini WS, Fontenelle LF, Almeida APSC. Qual o papel da Atenção Primária à Saúde diante da pandemia provocada pela COVID-19?Epidemiol. Serv. Saúde. 2020; 29(2):e2020166. Disponível em: http://www.scielo.br/scielo. php?script=sci_arttext\&pid=S2237-96222020000200903\&lng=en\&nrm=iso.

14. Ferguson NM, Laydon D, Nedjati-Gilani G, Imai N, Ainslie K, Baguelin M, Bhatia S, Boonyasiri A, Cucunubá Z, Cuomo-Dannenburg G, Dighe A. Impact of non-pharmaceutical interventions (NPIs) to reduce COVID-19 mortality and healthcare demand. Imperial College COVID-19 Response Team, London, Março, 16. Disponível em:https://spiral. imperial.ac.uk:8443/bitstream/10044/1/77482/14/2020-03-16-COVID19-Report-9.pdf.

15. Brooks SK, Webster RK, Smith LE, Woodland L, Wessely S, Greenberg N, Rubin GJ. The psychological impact of quarantine and how to reduce it: rapid review of the evidence. The Lancet. 2020; 395(10227):912-920. Elsevier BV. Disponívelem: https://www.thelancet.com/action/showPdf?pii=S0140-6736\%2820\%2930460-8.

16. Mook M, Mello PMVC. Pandemia COVID-19. Rev. bras. ter. Intensiva. 2020;32(1):1.

17. Raiol RA. Praticar exercícios físicos é fundamental para a saúde física e mental durante a Pandemia da COVID-19. Brazilian Journal Of Health Review. 2020;3(2):2804-2813. Disponível em: http://www.brazilianjournals.com/index.php/ BJHR/article/view/8463/7298.

18. Bao Y, Sun Y, Meng S, Shi J, Lu L. 2019-nCoV epidemic: address mental health care to empower society. The Lancet. 2020;395(10224):37-38. Disponível em: https://www.thelancet.com/pdfs/journals/lancet/PIIS0140-6736(20)30309-3.pdf.

19. Goyal K, Chauhan P, Chhikara K, Gupta P, Singh MP. Fear of COVID 2019: First Suicidal Case in India!. Asian Journal of Psychiatry. 2020;49:101989-101989. Disponível em: https://reader.elsevier.com/reader/sd/pii/S1876201820300976?token=1420C7BF03113337BF7DFCF0DEE5D1B8AD80A76D7525C18226B35664AD1782B79A664DBBBF979C0D85A55324BF491907.

20. Rios AFM, Lira LSSP, Reis IM, Silva GA. Atenção Primária à saúde frente à Covid-19 em um centro de saúde. Enferm. Foco. 2020;11(1)Especial: 246-251.

21. Daumas RP, Silva GA, Tasca R, Leite IC, Brasil P, Greco DB, Grabois V, Campos GWS. O papel da atenção primária na rede de atenção à saúde no Brasil: limites e possibilidades no enfrentamento da COVID-19. Cad. Saúde Pública. 2020;36(6):e00104120.

22. Greenhalgh T, Koh GCH, Car J. Covid-19: avaliação remota em Atenção Primária à Saúde. RevBrasMedFam Comunidade 2020;15:2461.

23. Brasil. Ministério da Saúde. Portaria MS/GM no 467, de 20 de março de 2020. Dispõe, em caráter excepcional e temporário, sobre as ações de Telemedicina, com o objetivo de regulamentar e operacionalizar as medidas de enfrentamento da emergência de saúde pública de importância internacional previstas no art. $3^{\circ}$ da Lei $n^{\circ} 13.979$, de 6 de fevereiro de 2020, decorrente da epidemia de COVID-19 [Internet]. Diário Oficial da União, Brasília (DF), 2020 mar 23 [citado 2020 abr 14];Seção 1:Extra. Disponível em: http://www.in.gov. br/en/web/dou/-/portaria-n-467-de-20-demarcode-2020-249312996. 